\title{
Isometric embeddings of half-cube graphs in half-spin Grassmannians
}

\author{
Mark Pankov \\ Department of Mathematics and Computer Science \\ University of Warmia and Mazury \\ Olsztyn, Poland \\ pankov@matman.uwm.edu.pl
}

Submitted: Feb 12, 2014; Accepted: Sep 22, 2014; Published: Oct 2, 2014

Mathematics Subject Classifications: 51A50, 51E24

\begin{abstract}
Let $\Pi$ be a polar space of type $D_{n}$. Denote by $\mathcal{G}_{\delta}(\Pi), \delta \in\{+,-\}$ the associated half-spin Grassmannians and write $\Gamma_{\delta}(\Pi)$ for the corresponding half-spin Grassmann graphs. In the case when $n \geqslant 4$ is even, the apartments of $\mathcal{G}_{\delta}(\Pi)$ will be characterized as the images of isometric embeddings of the half-cube graph $\frac{1}{2} H_{n}$ in $\Gamma_{\delta}(\Pi)$. As an application, we describe all isometric embeddings of $\Gamma_{\delta}(\Pi)$ in the half-spin Grassmann graphs associated to a polar space of type $\mathrm{D}_{n^{\prime}}$ under the assumption that $n \geqslant 6$ is even.
\end{abstract}

Keywords: half-cube graph; half-spin Grassmann graph; isometric embedding.

\section{Introduction}

In the present paper we continue to discuss the problem of metric characterization of apartments in building Grassmannians $[11,12]$. This problem is connected to the results obtained in $[3,4,8,9,13]$.

By [17], a building is a simplicial complex $\Delta$ containing a family of subcomplexes called apartments and characterized by some properties. All apartments are isomorphic to a certain Coxeter complex, i.e. the simplicial complex associated to a Coxeter system, which defines the type of the building. We suppose that our building is spherical, i.e. the associated Coxeter system is finite. Maximal simplices of $\Delta$ are said to be chambers. They have the same finite cardinality $n$ called the rank of $\Delta$. The vertex set of $\Delta$ can be labeled by the nodes of the diagram corresponding to the associated Coxeter system (such labeling is unique up to a permutation on the set of nodes). Every set consisting of all vertices labeled by the same node is said to be a Grassmannian (more general objects are 
investigated in [14]). The intersection of every chamber and every Grassmannian is a single vertex. Thus the vertex set of $\Delta$ is decomposed in precisely $n$ distinct Grassmannians. The intersections of apartments with one of the building Grassmannians are called apartments in this Grassmannian.

Let $\mathcal{G}$ be one of the building Grassmannians. We say that $a, b \in \mathcal{G}$ are adjacent if there is a simplex $P$ such that $P \cup\{a\}$ and $P \cup\{b\}$ are chambers. Consider the associated Grassmann graph $\Gamma$, i.e. the graph whose vertex set is $\mathcal{G}$ and whose edges are pairs of adjacent vertices. Suppose that $\mathcal{A}$ is an apartment of $\mathcal{G}$ and denote by $\Gamma_{\mathcal{A}}$ the restriction of the graph $\Gamma$ to $\mathcal{A}$. We want to distinguish all cases such that the image of every isometric embedding of $\Gamma_{\mathcal{A}}$ in $\Gamma$ is an apartment of $\mathcal{G}$. Note that in some cases this does not hold $[2,11]$.

By [17], there are the following seven types of irreducible thick spherical buildings of rank $\geqslant 3$ : three classical types $\mathrm{A}_{n}, \mathrm{~B}_{n}=\mathrm{C}_{n}, \mathrm{D}_{n}$ and four exceptional types $\mathrm{F}_{4}, \mathrm{E}_{i}, i=$ $6,7,8$.

Every building of type $\mathrm{A}_{n-1}$ is the flag complex of a certain $n$-dimensional vector space $V$ (over a division ring). The associated Grassmannians are $\mathcal{G}_{k}(V)$, where $k \in$ $\{1, \ldots, n-1\}$. Each $\mathcal{G}_{k}(V)$ consists of all $k$-dimensional subspaces of $V$ and two elements of $\mathcal{G}_{k}(V)$ are adjacent if their intersection is $(k-1)$-dimensional. The corresponding Grassmann graph is denoted by $\Gamma_{k}(V)$. The case when $k=1, n-1$ is trivial - any two distinct vertices of $\Gamma_{k}(V)$ are adjacent. Every apartment of $\mathcal{G}_{k}(V)$ is defined by a certain base of $V$ : it consists of all $k$-dimensional subspaces spanned by subsets of this base. All apartments of $\mathcal{G}_{k}(V)$ are the images of isometric embeddings of the Johnson graph $J(n, k)$ in $\Gamma_{k}(V)$. However, the image of every isometric embedding of $J(n, k)$ in $\Gamma_{k}(V)$ is an apartment if and only if $n=2 k[11]$.

All buildings of types $C_{n}$ and $D_{n}$ are defined by polar spaces. Every building of type $C_{n}$ is the flag complex formed by singular subspaces of a rank $n$ polar space. The associated Grassmannians are the polar Grassmannians consisting of singular subspaces of the same dimension. The polar Grassmannian whose elements are maximal singular subspaces is called the dual polar space and the associated Grassmann graph is known as the dual polar graph. By [12], the apartments in the dual polar space can be characterized as the images of isometric embeddings of the $n$-dimensional hypercube graph $H_{n}$ in the dual polar graph.

Every building of type $\mathrm{D}_{n}$ can be obtained from a polar space of type $\mathrm{D}_{n}$. This construction is known as the oriflamme complex. The Grassmannians of this building are some of the polar Grassmannians and so-called half-spin Grassmannians. The Grassmann graphs associated to the half-spin Grassmannians are called the half-spin Grassmann graphs. In this paper we show that the apartments of the half-spin Grassmannians can be characterized as the images of isometric embeddings of the half-cube graph $\frac{1}{2} H_{n}$ in the half-spin Grassmann graphs if $n \geqslant 4$ is even (Theorem 2). In the case when $n$ is odd, we conjecture the existence of isometric embeddings of $\frac{1}{2} H_{n}$ in the half-spin Grassmann graphs whose images are not apartments (Section 6). As an application of the main result, we describe all isometric embeddings of the half-spin Grassmann graphs of a polar space of type $\mathrm{D}_{n}$, where $n \geqslant 6$ is even, in the half-spin Grassmann graphs associated to a polar 
space of type $\mathrm{D}_{n^{\prime}}$ (Theorem 4).

Note that in [3] apartments in Grassmannians of finite-dimensional vector spaces, dual polar spaces and half-spin Grassmannians were characterized in terms of independent subsets in the associated partial linear spaces. Some more general results can be found in $[8,9]$. See [4] for a survey.

\section{Basic definitions and constructions}

\subsection{Graphs}

The distance between two vertices in a connected graph is the smallest number $i$ such that there is a path of length $i$ (a path consisting of $i$ edges) connecting these vertices. A path connecting two vertices is said to be a geodesic if the number of edges in this path is equal to the distance between the vertices. The maximum of all distances between vertices in a graph is called the diameter of the graph. In the case when the diameter is finite, two vertices are said to be opposite if the distance between them is equal to the diameter.

A subset in the vertex set of a graph is called a clique if any two distinct elements of this subset are adjacent vertices. Maximal cliques exist and every clique is contained in a certain maximal clique. For finite graph this is trivial and we use Zorn lemma for infinite graphs.

An injective mapping of the vertex set of a graph $\Gamma$ to the vertex set of a graph $\Gamma^{\prime}$ is an embedding of $\Gamma$ in $\Gamma^{\prime}$ if it transfers adjacent vertices to adjacent vertices and non-adjacent vertices to non-adjacent vertices. Every surjective embedding is an isomorphism between the graphs. An embedding is said to be isometric if it preserves the distance between vertices.

\subsection{Hypercube and half-cube graphs}

The vertex set of the $n$-dimensional hypercube graph $H_{n}$ is formed by all sequences $\left(a_{1}, \ldots, a_{n}\right)$, where each $a_{i}$ is equal to 0 or 1 . For any two such sequences $a=\left(a_{1}, \ldots, a_{n}\right)$ and $b=\left(b_{1}, \ldots, b_{n}\right)$ we define the distance

$$
d(a, b):=\left|a_{1}-b_{1}\right|+\cdots+\left|a_{n}-b_{n}\right| .
$$

The sequences $a$ and $b$ are adjacent vertices of $H_{n}$ if $d(a, b)=1$. The graph $H_{n}$ is connected, $d$ is the distance on $H_{n}$ and the diameter of $H_{n}$ is equal to $n$.

Lemma 1. For every vertex of $H_{n}$ there is unique vertex of $H_{n}$ opposite to this vertex. If $v$ and $w$ are opposite vertices of $H_{n}$ then every vertex of $H_{n}$ belongs to a geodesic connecting $v$ and $w$.

Proof. Easy verification. 
Remark 1. Consider the (2n)-element set $J=\{ \pm 1, \ldots, \pm n\}$. A subset $I \subset J$ is said to be singular if for every $i \in I$ we have $-i \notin I$. Every maximal singular subset consists of $n$ elements. The hypercube graph $H_{n}$ can be defined as the graph whose vertex set is formed by all maximal singular subsets. Two such subsets are adjacent vertices of $H_{n}$ if their intersection consists of $n-1$ elements.

The vertex set of $H_{n}$ can be uniquely decomposed in the sum of two disjoint subsets $A_{+}$and $A_{-}$such that the distance between any two elements of $A_{\delta}, \delta \in\{+,-\}$ is even and the distance between every element of $A_{+}$and every element of $A_{-}$is odd. Consider the graph $H_{\delta}, \delta \in\{+,-\}$ whose vertex set is $A_{\delta}$ and two vertices of $H_{\delta}$ are adjacent if the distance between them in $H_{n}$ is equal to 2. The graphs $H_{+}$and $H_{-}$are isomorphic. Each of these graphs is called the $n$-dimensional half-cube graph and denoted by $\frac{1}{2} H_{n}$.

The graph $\frac{1}{2} H_{n}$ is connected. If $v$ and $u$ are vertices of $\frac{1}{2} H_{n}$ then the distance between them is equal to $\frac{d(v, u)}{2}$. The diameter of $\frac{1}{2} H_{n}$ is equal to $\frac{n}{2}$ or $\frac{n-1}{2}$ if $n$ is even or odd, respectively.

There is the following analogue of Lemma 1.

Lemma 2. Suppose that $n$ is even. For every vertex of $\frac{1}{2} H_{n}$ there is a unique vertex of $\frac{1}{2} H_{n}$ opposite to this vertex. If $v$ and $w$ are opposite vertices of $\frac{1}{2} H_{n}$ then every vertex of $\frac{1}{2} H_{n}$ belongs to a geodesic connecting $v$ and $w$.

Proof. Easy verification.

If $n$ is odd then the above statement fails and for every vertex of $\frac{1}{2} H_{n}$ there are precisely $n$ vertices of $\frac{1}{2} H_{n}$ opposite to this vertex.

\subsection{Partial linear spaces}

Consider a non-empty set $P$ whose elements are called points and a family $\mathcal{L}$ of proper subsets of $P$ called lines. We say that two or more points are collinear if there is a line containing all of them. Suppose that the pair $\Pi=(P, \mathcal{L})$ is a partial linear space, i.e. the following axioms hold:

- every line contains at least two points and every point belongs to a line,

- for any distinct collinear points $p, q \in P$ there is precisely one line $p q$ containing them.

A subset $S \subset P$ is a subspace of $\Pi$ if for any distinct collinear points $p, q \in S$ the line $p q$ is contained in $S$. A subspace is said to be singular if any two distinct points of the subspace are collinear (by the definition, the empty set and a single point are singular subspaces). Using Zorn lemma, we establish the existence of maximal singular subspaces and the fact that every singular subspace is contained in a certain maximal singular subspace.

For every subset $X \subset P$ the minimal subspace containing $X$, i.e. the intersection of all subspaces containing $X$, is called spanned by $X$ and denoted by $\langle X\rangle$. We say that $X$ is independent if the subspace $\langle X\rangle$ cannot be spanned by a proper subset of $X$. 
Let $S$ be a subspace of $\Pi$ (possible $S=P$ ). An independent subset $X \subset S$ is a base of $S$ if $\langle X\rangle=S$. The dimension of $S$ is the smallest cardinality $\alpha$ such that $S$ has a base of cardinality $\alpha+1$. The dimension of the empty set and a single point is equal to -1 and 0 (respectively), lines are 1-dimensional subspaces. Every 2-dimensional singular subspace will be called a plane.

Two partial linear spaces $\Pi=(P, \mathcal{L})$ and $\Pi^{\prime}=\left(P^{\prime}, \mathcal{L}^{\prime}\right)$ are isomorphic if there is a bijection $f: P \rightarrow P^{\prime}$ satisfying $f(\mathcal{L})=\mathcal{L}^{\prime}$. Every such bijection is called a collineation of $\Pi$ to $\Pi^{\prime}$.

\subsection{Polar spaces}

By $[1,10,15,16]$, a polar space is a partial linear space $\Pi=(P, \mathcal{L})$ satisfying the following axioms:

- every line contains at least three points,

- there is no point collinear to all points,

- if $p \in P$ and $L \in \mathcal{L}$ then $p$ is collinear to one or all points of the line $L$,

- any sequence of distinct mutually incident singular subspaces is finite.

If our polar space contains a singular subspace whose dimension is not less than 2 then all maximal singular subspaces of $\Pi$ are projective spaces of the same dimension $n \geqslant 2$ and the number $n+1$ is called the rank of $\Pi$.

The collinearity relation of $\Pi$ will be denoted by $\perp$. For points $p, q \in P$ we write $p \perp q$ if $p$ is collinear to $q$ and $p \not \perp q$ otherwise. Moreover, if $X, Y \subset P$ then $X \perp Y$ means that every point of $X$ is collinear to all points of $Y$. If $X \perp X$ then the subspace $\langle X\rangle$ is singular.

For every polar space of rank $n$ one of the following possibilities is realized:

- type $\mathrm{C}_{n}$ - every $(n-2)$-dimensional singular subspace is contained in at least three maximal singular subspaces,

- type $\mathrm{D}_{n}$ - every $(n-2)$-dimensional singular subspace is contained in precisely two maximal singular subspaces.

All polar spaces of rank $\geqslant 3$ were described by J. Tits [17]. We will focus our attention on polar spaces of type $\mathrm{D}_{n}, n \geqslant 4$.

Example 1. Let $V$ be a $(2 n)$-dimensional vector space over a field. Suppose that the characteristic of this field is not equal to 2 and consider a non-degenerate symmetric bilinear form on $V$ such that maximal totally isotropic subspaces are $n$-dimensional. The associated polar space whose points are 1-dimensional isotropic subspaces and whose lines are defined by 2-dimensional totally isotropic subspaces (the line corresponding to a 2dimensional totally isotropic subspace $S$ consists of all 1-dimensional subspaces of $S$ ) is 
of type $\mathrm{D}_{n}$. In the case when the characteristic of the field is equal to 2 , we consider a non-defect quadratic form on $V$ such that maximal singular subspaces are $n$-dimensional. The associated polar space (the points are 1-dimensional singular subspaces and the lines are defined by 2-dimensional singular subspaces) is also of type $\mathrm{D}_{n}$.

It follows from Tits's description of polar spaces that every polar space of type $\mathrm{D}_{n}$, $n \geqslant 4$ is isomorphic to one of the polar spaces presented in Example 1.

\subsection{Polar Grassmannians}

Let $\Pi=(P, \mathcal{L})$ be a polar space of rank $n$. For every $k \in\{0,1, \ldots, n-1\}$ we denote by $\mathcal{G}_{k}(\Pi)$ the polar Grassmannian consisting of all $k$-dimensional singular subspaces of $\Pi$; in particular, $\mathcal{G}_{n-1}(\Pi)$ is formed by maximal singular subspaces.

The dual polar graph $\Gamma_{n-1}(\Pi)$ is the graph whose vertex set is $\mathcal{G}_{n-1}(\Pi)$ and two distinct elements of $\mathcal{G}_{n-1}(\Pi)$ are adjacent vertices of $\Gamma_{n-1}(\Pi)$ if their intersection is an $(n-2)$ dimensional singular subspace. The graph $\Gamma_{n-1}(\Pi)$ is connected and the distance $d(S, U)$ between $S, U \in \mathcal{G}_{n-1}(\Pi)$ is equal to

$$
n-1-\operatorname{dim}(S \cap U) .
$$

The diameter of $\Gamma_{n-1}(\Pi)$ is equal to $n$ and two vertices of $\Gamma_{n-1}(\Pi)$ are opposite if and only if they are disjoint singular subspaces.

Let $S$ and $U$ be singular subspaces of $\Pi$ such that $S \subset U$. If

$$
\operatorname{dim} S<k<\operatorname{dim} U
$$

then we write $[S, U]_{k}$ for the set of all $X \in \mathcal{G}_{k}(\Pi)$ satisfying $S \subset X \subset U$. In the case when $S=\emptyset$, this set will be denoted by $\langle U]_{k}$.

Let $M$ be an $m$-dimensional singular subspace of $\Pi$. For every natural $k>m$ we denote by $[M\rangle_{k}$ the set of all elements of $\mathcal{G}_{k}(\Pi)$ containing $M$. Now suppose that $m<n-2$. For every $N \in \mathcal{G}_{m+2}(\Pi)$ containing $M$ the set $[M, N]_{m+1}$ is called a line of $[M\rangle_{m+1}$. The set $[M\rangle_{m+1}$ together with the family of all such lines is a polar space of rank $n-m-1[10$, Lemma 4.4]. This polar space will be denoted by $\Pi_{M}$. Every maximal singular subspace of $\Pi_{M}$ is of type $[M, U]_{m+1}$, where $U$ is a maximal singular subspace of $\Pi$ containing $M$. Thus $[M\rangle_{n-1}$ can be naturally identified with $\mathcal{G}_{n-m-2}\left(\Pi_{M}\right)$ and the dual polar graph $\Gamma_{n-m-2}\left(\Pi_{M}\right)$ is the restriction of the graph $\Gamma_{n-1}(\Pi)$ to $[M\rangle_{n-1}$. In the case when $\Pi$ is a polar space of type $\mathrm{D}_{n}$, the polar space $\Pi_{M}$ is of type $\mathrm{D}_{n-m-1}$.

\subsection{Half-spin Grassmannians}

Let $\Pi=(P, \mathcal{L})$ be a polar space of type $\mathrm{D}_{n}$. Then $\mathcal{G}_{n-1}(\Pi)$ can be uniquely decomposed in the sum of two disjoint subsets, we denote them by $\mathcal{G}_{+}(\Pi)$ and $\mathcal{G}_{-}(\Pi)$, such that the distance between any two elements of $\mathcal{G}_{\delta}(\Pi), \delta \in\{+,-\}$ in the dual polar graph $\Gamma_{n-1}(\Pi)$ is even and the same distance between any $S \in \mathcal{G}_{\delta}(\Pi)$ and $U \in \mathcal{G}_{-\delta}(\Pi)$ is odd (we write 
$-\delta$ for the complement of $\delta$ in the set $\{+,-\})$. These subsets are called the half-spin Grassmannians of $\Pi$.

The half-spin Grassmann graph $\Gamma_{\delta}(\Pi), \delta \in\{+,-\}$ is the graph whose vertex set is $\mathcal{G}_{\delta}(\Pi)$ and two elements of $\mathcal{G}_{\delta}(\Pi)$ are adjacent vertices of $\Gamma_{\delta}(\Pi)$ if their intersection is an $(n-3)$-dimensional singular subspace, i.e. the distance between the corresponding vertices of $\Gamma_{n-1}(\Pi)$ is equal to 2 .

This graph is connected and the distance $d_{\delta}(S, U)$ between $S, U \in \mathcal{G}_{\delta}(\Pi)$ in $\Gamma_{\delta}(\Pi)$ is equal to $\frac{d(S, U)}{2}$. If $n$ is even then the diameter of $\Gamma_{\delta}(\Pi)$ is equal to $\frac{n}{2}$ and two vertices are opposite if and only if they are disjoint singular subspaces. In the case when $n$ is odd, the diameter is equal to $\frac{n-1}{2}$ and two vertices are opposite if and only if their intersection is a single point.

Remark 2. If a polar space is defined by a non-degenerate symmetric bilinear form $\Omega$ (see Example 1) then their maximal singular subspaces can be identified with the maximal totally isotropic subspaces of $\Omega$ and the half-spin Grassmannians are the orbits of the action of the orthogonal group $\mathrm{O}^{+}(\Omega)$ on the set of all maximal totally isotropic subspaces. Every element of $\mathrm{O}(\Omega) \backslash \mathrm{O}^{+}(\Omega)$ transfers one half-spin Grassmannian to the other and it induces an isomorphism between the half-spin Grassmann graphs. The same holds for the polar spaces defined by quadratic forms.

The latter remark shows that the graphs $\Gamma_{+}(\Pi)$ and $\Gamma_{-}(\Pi)$ are isomorphic. For $n=2,3$ any two distinct vertices of $\Gamma_{\delta}(\Pi)$ are adjacent and we will always suppose that $n \geqslant 4$.

Let $M$ be an $m$-dimensional singular subspace of $\Pi$ and $m<n-2$. Denote by $[M\rangle_{\delta}$ the set of all elements of $\mathcal{G}_{\delta}(\Pi)$ containing $M$. In the case when $m=n-3$, this set is called a line of $\mathcal{G}_{\delta}(\Pi)$. Any two distinct elements of such a line are adjacent vertices of $\Gamma_{\delta}(\Pi)$. If $m<n-3$ then $\Pi_{M}$ is a polar space of type $\mathrm{D}_{n-m-1}$ whose half-spin Grassmannian $\mathcal{G}_{\delta}\left(\Pi_{M}\right)$ can be naturally identified with $[M\rangle_{\delta}$ and the half-spin Grassmann graph $\Gamma_{\delta}\left(\Pi_{M}\right)$ is the restriction of the graph $\Gamma_{\delta}(\Pi)$ to $[M\rangle_{\delta}$.

\section{Main result}

Let $\Pi=(P, \mathcal{L})$ be a polar space of rank $n$. A set $\mathcal{F}$ consisting of $2 n$ distinct points $p_{1}, \ldots, p_{2 n} \in P$ is called a frame of $\Pi$ if for every $i \in\{1 \ldots, 2 n\}$ there exists unique $\sigma(i) \in\{1 \ldots, 2 n\}$ such that $p_{i} \not \perp p_{\sigma(i)}$. This is an independent subset and any $k$ distinct mutually collinear points of $\mathcal{F}$ span a $(k-1)$-dimensional singular subspace. Denote by $\mathcal{A}$ the set consisting of all maximal singular subspaces spanned by subsets of $\mathcal{F}$, i.e. all subspaces of type

$$
\left\langle p_{i_{1}}, \ldots, p_{i_{n}}\right\rangle
$$

such that

$$
\left\{i_{1}, \ldots, i_{n}\right\} \cap\left\{\sigma\left(i_{1}\right), \ldots, \sigma\left(i_{n}\right)\right\}=\emptyset .
$$

This set is the apartment of $\mathcal{G}_{n-1}(\Pi)$ associated to the frame $\mathcal{F}$. It follows from Remark 1 that $\mathcal{A}$ is the image of an isometric embedding of the $n$-dimensional hypercube graph $H_{n}$ 
in the dual polar graph $\Gamma_{n-1}(\Pi)$. If $M$ is an $(n-m-1)$-dimensional singular subspace of $\Pi$ then $\Pi_{M}$ is a polar space of rank $m$ and every apartment of $\mathcal{G}_{m-1}\left(\Pi_{M}\right)=[M\rangle_{n-1}$ is the image of an isometric embedding of $H_{m}$ in $\Gamma_{n-1}(\Pi)$.

Theorem 1 ([12]). The image of every isometric embedding of the $m$-dimensional hypercube graph $H_{m}, m \leqslant n$ in the dual polar graph $\Gamma_{n-1}(\Pi)$ is an apartment of $\mathcal{G}_{m-1}\left(\Pi_{M}\right)$, where $M$ is an $(n-m-1)$-dimensional singular subspace of $\Pi$. In particular, the image of every isometric embedding of $H_{n}$ in $\Gamma_{n-1}(\Pi)$ is an apartment of $\mathcal{G}_{n-1}(\Pi)$.

Now suppose that $\Pi$ is a polar space of type $D_{n}$ and $\delta \in\{+,-\}$. The intersection

$$
\mathcal{A}_{\delta}:=\mathcal{A} \cap \mathcal{G}_{\delta}(\Pi)
$$

is the apartment of the half-spin Grassmannian $\mathcal{G}_{\delta}(\Pi)$ associated to the frame $\mathcal{F}$. This is the image of an isometric embedding of the half-cube graph $\frac{1}{2} H_{n}$ in the half-spin Grassmann graph $\Gamma_{\delta}(\Pi)$ (this easily follows from the fact that $\mathcal{A}$ is the image of an isometric embedding of $H_{n}$ in $\left.\Gamma_{n-1}(\Pi)\right)$. If $M$ is an $(n-m-1)$-dimensional singular subspace of $\Pi$ then $\Pi_{M}$ is a polar space of type $\mathrm{D}_{m}$ and every apartment of $\mathcal{G}_{\delta}\left(\Pi_{M}\right)=[M\rangle_{\delta}$ is the image of an isometric embedding of $\frac{1}{2} H_{m}$ in $\Gamma_{\delta}(\Pi)$.

Example 2. Suppose that $n=4$. Then $\mathcal{G}_{\delta}(\Pi)$ together with the family of all lines is a polar space of type $\mathrm{D}_{4}[10$, Proposition 4.23$]$. This polar space will be denoted by $\Pi_{\delta}$. Two distinct points of $\Pi_{\delta}$ are collinear if and only if they are adjacent vertices of $\Gamma_{\delta}(\Pi)$. The graph $\frac{1}{2} H_{4}$ consists of 8 vertices and for every vertex there is precisely one vertex non-adjacent to it. This means that the frames of $\Pi_{\delta}$ can be characterized as the images of embeddings of $\frac{1}{2} H_{4}$ in $\Gamma_{\delta}(\Pi)$ (every such embedding is isometric, since both graphs are of diameter 2). By [10, Corollary 4.4] (see also [3]), the family of all apartments of $\mathcal{G}_{\delta}(\Pi)$ coincides with the family of all frames of $\Pi_{\delta}$. So, the image of every embedding of $\frac{1}{2} H_{4}$ in $\Gamma_{\delta}(\Pi)$ is an apartment of $\mathcal{G}_{\delta}(\Pi)$.

The main result of the present paper is the following.

Theorem 2. Suppose that $\Pi$ is a polar space of type $\mathrm{D}_{n}$. If $m$ is an even integer satisfying $4 \leqslant m \leqslant n$ then the image of every isometric embedding of the $m$-dimensional half-cube graph $\frac{1}{2} H_{m}$ in the half-spin Grassmann graph $\Gamma_{\delta}(\Pi), \delta \in\{+,-\}$ is an apartment of $\mathcal{G}_{\delta}\left(\Pi_{M}\right)$, where $M$ is an $(n-m-1)$-dimensional singular subspace of $\Pi$. In particular, if $n$ is even then the image of every isometric embedding of $\frac{1}{2} H_{n}$ in $\Gamma_{\delta}(\Pi)$ is an apartment of $\mathcal{G}_{\delta}(\Pi)$.

In Section 7, we apply Theorem 2 to isometric embeddings of half-spin Grassmann graphs.

\section{Cliques}

\subsection{Maximal cliques of half-spin Grassmannian graphs}

Let $\Pi=(P, \mathcal{L})$ be a polar space of type $\mathrm{D}_{n}$ and $\delta \in\{+,-\}$. By [10, Subsection 4.5.2], there are precisely the following two types of maximal cliques of $\Gamma_{\delta}(\Pi)$ : 
- The star $[M\rangle_{\delta}, M \in \mathcal{G}_{n-4}(\Pi)$ (if two distinct elements of $\mathcal{G}_{\delta}(\Pi)$ contain $M$ then their intersection is $(n-3)$-dimensional and they are adjacent vertices of $\left.\Gamma_{\delta}(\Pi)\right)$. The star $[M\rangle_{\delta}$ together with all lines of $\mathcal{G}_{\delta}(\Pi)$ contained in $[M\rangle_{\delta}$ is a 3-dimensional projective space.

- The special subspace $[U]_{\delta}, U \in \mathcal{G}_{-\delta}(\Pi)$ formed by all elements of $\mathcal{G}_{\delta}(\Pi)$ intersecting $U$ in $(n-2)$-dimensional singular subspaces, i.e. all vertices of $\Gamma_{n-1}(\Pi)$ adjacent to $U$. The special subspace $[U]_{\delta}$ together with all lines of $\mathcal{G}_{\delta}(\Pi)$ contained in $[U]_{\delta}$ is an $(n-1)$-dimensional projective space.

Recall the following facts concerning the intersection of two distinct maximal cliques:

- The intersection of two distinct stars $[M\rangle_{\delta}$ and $\left[M^{\prime}\right\rangle_{\delta}$ is empty or a single vertex or a line. The second possibility is not realized if $n=4$. The intersection is a line if and only if $M$ and $M^{\prime}$ span an $(n-3)$-dimensional singular subspace.

- The intersection of two distinct special subspaces is empty or a line. The second possibility is realized if and only if the associated elements of $\mathcal{G}_{-\delta}(\Pi)$ are adjacent vertices of $\Gamma_{-\delta}(\Pi)$.

- The dimension of the intersection of a star $[M\rangle_{\delta}$ and a special subspace $[U]_{\delta}$ is not greater than 2. This intersection is a plane if and only if $M$ is contained in $U$.

Therefore, the dimension of the intersection of two distinct maximal cliques of $\Gamma_{\delta}(\Pi)$ is not greater than 2. If this intersection is a plane then one of the cliques is a star and the other is a special subspace.

Lemma 3. For any distinct maximal cliques $\mathcal{C}$ and $\mathcal{C}^{\prime}$ in $\Gamma_{\delta}(\Pi)$ there is a sequence of maximal cliques

$$
\mathcal{C}=\mathcal{C}_{0}, \mathcal{C}_{1}, \ldots, \mathcal{C}_{i}=\mathcal{C}^{\prime}
$$

such that $\mathcal{C}_{j-1} \cap \mathcal{C}_{j}$ is a plane for every $j \in\{1, \ldots, i\}$. The cliques $\mathcal{C}$ and $\mathcal{C}^{\prime}$ are of the same type (both are stars or both are special subspaces) if and only if $i$ is even.

Proof. Consider the elements of $\mathcal{G}_{n-4}(\Pi) \cup \mathcal{G}_{-\delta}(\Pi)$ associated to $\mathcal{C}$ and $\mathcal{C}^{\prime}$. There is a frame $\mathcal{F}$ such that these singular subspaces are spanned by subsets of $\mathcal{F}$ (this follows from one of the basic building properties [17]). We construct the required sequence of maximal cliques such that the corresponding elements of $\mathcal{G}_{n-4}(\Pi) \cup \mathcal{G}_{-\delta}(\Pi)$ are spanned by subsets of the frame $\mathcal{F}$.

\subsection{Maximal cliques of half-cube graphs}

As in the previous subsection, we suppose that $\Pi=(P, \mathcal{L})$ is a polar space of type $\mathrm{D}_{n}$. Let $\delta \in\{+,-\}$ and let $\mathcal{A}$ be the apartment of $\mathcal{G}_{\delta}(\Pi)$ defined by a frame $\mathcal{F}$.

The restriction of $\Gamma_{\delta}(\Pi)$ to $\mathcal{A}$ is isomorphic to the half-cube graph $\frac{1}{2} H_{n}$. Every clique of this restriction is a clique in $\Gamma_{\delta}(\Pi)$. Therefore, this graph has precisely the following two types of maximal cliques: 
- The star $\mathcal{A} \cap[S\rangle_{\delta}$, where $S \in \mathcal{G}_{n-4}(\Pi)$ is spanned by a subset of $\mathcal{F}$. This is a base of the projective space $[S\rangle_{\delta}$, i.e. it consists of 4 vertices.

- The special subset $\mathcal{A} \cap[U]_{\delta}$, where $U \in \mathcal{G}_{-\delta}(\Pi)$ is spanned by a subset of $\mathcal{F}$. This is a base of the projective space $[U]_{\delta}$. It consists of $n$ elements of $\mathcal{A}$ adjacent to $U$ in $\Gamma_{n-1}(\Pi)$.

The intersection of two distinct stars contains at most two vertices. This intersection is maximal if and only if the associated elements of $\mathcal{G}_{n-4}(\Pi)$ span an element of $\mathcal{G}_{n-3}(\Pi)$. Similarly, the intersection of two distinct special subsets contains at most two vertices and this intersection is maximal only in the case when the associated elements of $\mathcal{G}_{-\delta}(\Pi)$ are adjacent vertices of $\Gamma_{-\delta}(\Pi)$. The intersection of a star and a special subset contains at most three vertices. This intersection is maximal if and only if the associated elements of $\mathcal{G}_{n-4}(\Pi)$ and $\mathcal{G}_{-\delta}(\Pi)$ are incident. Therefore, the intersection of two distinct maximal cliques contains precisely three vertices if and only if one of these maximal cliques is a star and the other is a special subset. There is the following analogue of Lemma 3.

Lemma 4. For any distinct maximal cliques $\mathcal{C}$ and $\mathcal{C}^{\prime}$ there is a sequence of maximal cliques

$$
\mathcal{C}=\mathcal{C}_{0}, \mathcal{C}_{1}, \ldots, \mathcal{C}_{i}=\mathcal{C}^{\prime}
$$

such that $\left|\mathcal{C}_{j-1} \cap \mathcal{C}_{j}\right|=3$ for every $j \in\{1, \ldots, i\}$. The cliques $\mathcal{C}$ and $\mathcal{C}^{\prime}$ are of the same type if and only if $i$ is even.

Let $\mathcal{A}^{\prime}$ be another apartment of $\mathcal{G}_{\delta}(\Pi)$. Suppose that $h$ is an isomorphism between the restrictions of $\Gamma_{\delta}(\Pi)$ to $\mathcal{A}$ and $\mathcal{A}^{\prime}$. A star and a special subset are of the same cardinality only in the case when $n=4$. This means that $h$ sends stars to stars and special subsets to special subsets if $n>4$. If $n=4$ and there is a star whose image is a special subset then Lemma 4 implies that all stars go to special subsets and all special subsets go to stars.

In the next section we will use the following fact: if $h$ transfers stars to stars and special subsets to special subsets then for every $S \in \mathcal{G}_{k}(\Pi), k \in\{0, \ldots, n-2\}$ spanned by a subset of $\mathcal{F}$ there is $S^{\prime} \in \mathcal{G}_{k}(\Pi)$ spanned by a subset of the frame associated to $\mathcal{A}^{\prime}$ such that

$$
h\left(\mathcal{A} \cap[S\rangle_{\delta}\right)=\mathcal{A}^{\prime} \cap\left[S^{\prime}\right\rangle_{\delta}
$$

\section{Proof of Theorem 2}

\subsection{Reduction}

We show that the general case can be reduced to the case when $m=n$. Let $\Pi=(P, \mathcal{L})$ be a polar space of rank $n$.

Lemma 5 (Lemma 2 in [12]). If $X_{0}, \ldots, X_{i}$ is a geodesic in $\Gamma_{n-1}(\Pi)$ then

$$
X_{0} \cap X_{i} \subset X_{j}
$$

for every $j \in\{1, \ldots, i-1\}$. 
Now suppose that $\Pi$ is a polar space of type $\mathrm{D}_{n}$ and $\delta \in\{+,-\}$.

Lemma 6. If $Y_{0}, \ldots, Y_{i}$ is a geodesic in $\Gamma_{\delta}(\Pi)$ then

$$
Y_{0} \cap Y_{i} \subset Y_{j}
$$

for every $j \in\{1, \ldots, i-1\}$.

Proof. There exists a geodesic $X_{0}, \ldots, X_{2 i}$ in $\Gamma_{n-1}(\Pi)$ such that $X_{2 j}=Y_{j}$ for every $j \in$ $\{0, \ldots, i\}$. Lemma 5 gives the claim.

Lemma 7. If $m$ is an even integer not greater than $n$ then for every isometric embedding $f$ of $\frac{1}{2} H_{m}$ in $\Gamma_{\delta}(\Pi)$ there is an $(n-m-1)$-dimensional singular subspace $M$ such that the image of $f$ is contained in $[M\rangle_{\delta}$, in other words, $f$ can be considered as an isometric embedding of $\frac{1}{2} H_{m}$ in $\Gamma_{\delta}\left(\Pi_{M}\right)$, where $\Pi_{M}$ is the associated polar space of type $\mathrm{D}_{m}$.

Proof. Let $v$ and $w$ be opposite vertices of $\frac{1}{2} H_{m}$. Then

$$
m=2 d_{\delta}((f(v), f(w)))=d(f(v), f(w))=n-1-\operatorname{dim}(f(v) \cap f(w))
$$

which implies that

$$
M:=f(v) \cap f(w)
$$

is an $(n-m-1)$-dimensional singular subspace. Our statement is a simple consequence of Lemmas 2 and 6.

By Lemma 7, it is sufficient to prove Theorem 2 only in the case when $m=n$.

Remark 3. In the case when $m$ is odd, the graph $\frac{1}{2} H_{m}$ is more complicated (Subsection 2.2 ) and we cannot prove the latter statement.

\subsection{Proof of Theorem 2 for the case $m=n$}

Let $\Pi=(P, \mathcal{L})$ be a polar space of type $\mathrm{D}_{n}$ and let $\mathcal{F}=\left\{p_{1}, \ldots, p_{2 n}\right\}$ be a frame of $\Pi$. For every integer $k \in\{1, \ldots, n-1\}$ we denote by $\mathcal{A}_{k}$ the associated apartment of $\mathcal{G}_{k}(\Pi)$, i.e. the set formed by all $k$-dimensional singular subspaces of type

$$
\left\langle p_{i_{1}}, \ldots, p_{i_{k+1}}\right\rangle \text {. }
$$

Note that points $p_{i_{1}}, \ldots, p_{i_{k}}$ span a $(k-1)$-dimensional singular subspace if and only if

$$
\left\{i_{1}, \ldots, i_{k}\right\} \cap\left\{\sigma\left(i_{1}\right), \ldots, \sigma\left(i_{k}\right)\right\}=\emptyset,
$$

or equivalently, these points are mutually collinear. In this case, we will say that $i_{1}, \ldots, i_{k}$ form a singular set.

Let $\delta \in\{+,-\}$. Then

$$
\mathcal{A}:=\mathcal{A}_{n-1} \cap \mathcal{G}_{\delta}(\Pi)
$$


is the apartment of $\mathcal{G}_{\delta}(\Pi)$ associated to $\mathcal{F}$. For every singular set $\left\{i_{1}, \ldots, i_{k}\right\}$ we denote by $\mathcal{A}\left(i_{1}, \ldots, i_{k}\right)$ the set of all elements of $\mathcal{A}$ containing $p_{i_{1}}, \ldots, p_{i_{k}}$, i.e.

$$
\mathcal{A}\left(i_{1}, \ldots, i_{k}\right)=\mathcal{A} \cap[S\rangle_{\delta}
$$

where $S=\left\langle p_{i_{1}}, \ldots, p_{i_{k}}\right\rangle$.

It was noted in Subsection 4.2 that the restriction of $\Gamma_{\delta}(\Pi)$ to $\mathcal{A}$ is isomorphic to $\frac{1}{2} H_{n}$. Let $f: \mathcal{A} \rightarrow \mathcal{G}_{\delta}(\Pi)$ be an isometric embedding of $\frac{1}{2} H_{n}$ in $\Gamma_{\delta}(\Pi)$. Then

$$
\operatorname{dim}(X \cap Y)=\operatorname{dim}(f(X) \cap f(Y))
$$

for all $X, Y \in \mathcal{A}$.

Using induction we show that $\mathcal{X}:=f(\mathcal{A})$ is an apartment of $\mathcal{G}_{\delta}(\Pi)$ if $n$ is even. For $n=4$ this is true, see Example 2. Suppose that the statement holds for $n=2 l \geqslant 4$ and consider the case when $n=2(l+1) \geqslant 6$.

For every singular set $\left\{i_{1}, \ldots, i_{k}\right\}$ we define

$$
\mathcal{X}\left(i_{1}, \ldots, i_{k}\right):=f\left(\mathcal{A}\left(i_{1}, \ldots, i_{k}\right)\right) .
$$

If $i, j$ is a singular pair then the restriction of $\Gamma_{\delta}(\Pi)$ to $\mathcal{A}(i, j)$ is isomorphic to $\frac{1}{2} H_{n-2}$ and Lemma 7 implies the existence of a line $L_{i j}$ such that

$$
\mathcal{X}(i, j) \subset\left[L_{i j}\right\rangle_{\delta}
$$

Lemma 8. If $i, j$ and $s, t$ are singular pairs satisfying $\{i, j\} \cap\{s, t\}=\emptyset$ then

$$
L_{i j} \cap L_{s t}=\emptyset .
$$

Proof. We choose $X \in \mathcal{A}(i, j)$ and $Y \in \mathcal{A}(s, t)$ such that $X \cap Y=\emptyset$. Then

$$
f(X) \cap f(Y)=\emptyset .
$$

The statement follows from the fact that $f(X) \in \mathcal{X}(i, j)$ and $f(Y) \in \mathcal{X}(s, t)$ contain $L_{i j}$ and $L_{s t}$, respectively.

Let $i, j$ be a singular pair. Then $\Pi_{p_{i} p_{j}}$ is a polar space of type $\mathrm{D}_{n-2}$ and $\mathcal{A}(i, j)$ is an apartment of $\mathcal{G}_{\delta}\left(\Pi_{p_{i} p_{j}}\right)$. The inductive hypothesis implies that $\mathcal{X}(i, j)$ is an apartment of $\mathcal{G}_{\delta}\left(\Pi_{L_{i j}}\right)$. Our embedding induces an isomorphism between the restrictions of $\Gamma_{\delta}(\Pi)$ to $\mathcal{A}(i, j)$ and $\mathcal{X}(i, j)$ (they are isomorphic to $\frac{1}{2} H_{n-2}$ ) and, by Subsection 4.2, we have the following possibilities:

(1) $f$ transfers every star of $\mathcal{A}(i, j)$ to a star of $\mathcal{X}(i, j)$,

(2) $n=6$ and $f$ sends every star of $\mathcal{A}(i, j)$ to a special subset of $\mathcal{X}(i, j)$.

Lemma 9. The second possibility is not realized. 
Proof. Suppose that $n=6$ and $f$ transfers every star of $\mathcal{A}(i, j)$ to a special subset of $\mathcal{X}(i, j)$. We take any $t$ such that $i, j, t$ form a singular set. Then $\mathcal{A}(i, j, t)$ is a star of $\mathcal{A}(i, j)$ and, by our assumption, $\mathcal{X}(i, j, t)$ is a special subset of $\mathcal{X}(i, j)$. The latter implies that the intersection of all elements from $\mathcal{X}(i, j, t)$ coincides with $L_{i j}$.

On the other hand, $\mathcal{A}(i, j, t)$ is a star in $\mathcal{A}(i, t)$ and $\mathcal{A}(j, t)$. Hence $\mathcal{X}(i, j, t)$ is a special subset in $\mathcal{X}(i, t)$ and $\mathcal{X}(j, t)$. By the above arguments, the lines $L_{i t}$ and $L_{j t}$ both coincide with $L_{i j}$.

Now we take any $s \neq t$ such that $i, j, s$ form a singular set. Then $\mathcal{A}(i, j, s)$ is a star of $\mathcal{A}(i, j)$ and $\mathcal{X}(i, j, s)$ is a special subset of $\mathcal{X}(i, j)$. As above, we establish that $L_{i s}$ coincides with $L_{i j}$. Thus $L_{i s}=L_{j t}$ which contradicts Lemma 8.

Lemma 9 shows that $f$ transfers every star of $\mathcal{A}(i, j)$ to a star of $\mathcal{X}(i, j)$. Then, by Subsection 4.2 , for every $t$ such that $\{i, j, t\}$ is a singular set there is a plane $S_{i j}(t)$ containing $L_{i j}$ and such that $\mathcal{X}(i, j, t)$ consists of all elements of $\mathcal{X}(i, j)$ containing $S_{i j}(t)$. The intersection of all elements from $\mathcal{X}(i, j, t)$ coincides with $S_{i j}(t)$. Since

$$
\mathcal{A}(i, j, t)=\mathcal{A}(i, j) \cap \mathcal{A}(i, t) \cap \mathcal{A}(j, t),
$$

we have

$$
\mathcal{X}(i, j, t)=\mathcal{X}(i, j) \cap \mathcal{X}(i, t) \cap \mathcal{X}(j, t)
$$

and

$$
S_{i j}(t)=S_{i t}(j)=S_{j t}(i) .
$$

In what follows this plane will be denoted by $S_{i j t}$. The following fact is obvious.

Lemma 10. If $i, j$ is a singular pair then all $S_{i j t}$ with $t \notin\{i, j, \sigma(i), \sigma(j)\}$ form a frame of the polar space $\Pi_{L_{i j}}$ and $\mathcal{X}(i, j)$ is the apartment of $\mathcal{G}_{\delta}\left(\Pi_{L_{i j}}\right)$ associated to this frame.

The plane $S_{i j t}$ contains the lines $L_{i j}, L_{i t}, L_{j t}$. Hence for any pair of these lines one of the following possibilities is realized: the lines are coincident or they are distinct and span $S_{i j t}$.

Lemma 11. If $i, j, t$ form a singular set then the lines $L_{i j}, L_{i t}, L_{j t}$ are mutually distinct, i.e. any two of these lines span the plane $S_{i j t}$.

Proof. Suppose that $L_{i j}=L_{i t}$. Consider $X \in \mathcal{A}(i, j)$ and $Y \in \mathcal{A}(i, t)$ such that

$$
X \cap Y=p_{i} p_{s} \text { and } s \neq j, t .
$$

Then $X, Y \in \mathcal{A}(i, s)$ and $f(X), f(Y) \in \mathcal{X}(i, s)$. Since $f(X) \cap f(Y)$ is a line, we have

$$
f(X) \cap f(Y)=L_{i s} .
$$

On the other hand, $f(X) \in \mathcal{X}(i, j)$ contains $L_{i j}$ and $f(Y) \in \mathcal{X}(i, t)$ contains $L_{i t}$. This means that the line $L_{i j}=L_{i t}$ is contained in both $f(X)$ and $f(Y)$, i.e. it coincides with $L_{i s}$. Therefore, the lines $L_{i s}$ and $L_{j t}$ have a non-empty intersection $\left(L_{i s}\right.$ coincides with $L_{i j}$ and the latter line has a non-empty intersection with $L_{j t}$ ) which is impossible by Lemma 8 . 
Consider the graph $\Gamma_{1}(\Pi)$ whose vertex set is $\mathcal{G}_{1}(\Pi)$ and two lines are adjacent vertices of $\Gamma_{1}(\Pi)$ if they span a plane. By [10, Proposition 4.16], maximal cliques of this graph are of the following two types:

- the star $[p, U]_{1}$, where $p$ is a point of $U \in \mathcal{G}_{n-1}(\Pi)$;

- the top $\langle T]_{1}$, where $T \in \mathcal{G}_{2}(\Pi)$.

An easy verification shows that the restriction of the graph $\Gamma_{1}(\Pi)$ to $\mathcal{A}_{1}$ has precisely the following two types of maximal cliques:

- the star $\mathcal{A}_{1} \cap\left[p_{i}, U\right]_{1}$, where $U \in \mathcal{A}_{n-1}$ contains $p_{i}$;

- the top $\mathcal{A}_{1} \cap\langle T]_{1}$, where $T \in \mathcal{A}_{2}$.

Denote by $g$ the mapping of $\mathcal{A}_{1}$ to $\mathcal{G}_{1}(\Pi)$ sending every line $p_{i} p_{j}$ to the line $L_{i j}$. It follows from Lemma 11 that $g$ transfers adjacent vertices of $\Gamma_{1}(\Pi)$ to adjacent vertices of $\Gamma_{1}(\Pi)$. Then $g$ sends every star of $\mathcal{A}_{1}$ to a subset in a star or a top of $\mathcal{G}_{1}(\Pi)$. We cannot state that $g$ is injective, but its restriction to a star or a top of $\mathcal{A}_{1}$ is injective.

Lemma 12. Let $U \in \mathcal{A}$ and $p_{i} \in U$. Then

$$
g\left(\mathcal{A}_{1} \cap\left[p_{i}, U\right]_{1}\right)
$$

is contained in a star of $\mathcal{G}_{1}(\Pi)$.

Proof. Let $J$ be the set of all $j \neq i$ such that $p_{j} \in U$. Then $\mathcal{A}_{1} \cap\left[p_{i}, U\right]_{1}$ consists of all lines $p_{i} p_{j}$ with $j \in J$ and (1) is formed by all $L_{i j}$ with $j \in J$. Now we fix $j \in J$ and consider $f(U)$ as an element of $\mathcal{X}(i, j)$. Since $U$ belongs to $\mathcal{A}(i, j, t)$ for every $t \in J \backslash\{j\}$, the planes $S_{i j t}, t \in J \backslash\{j\}$ are contained in $f(U)$ and Lemma 10 implies that $f(U)$ is spanned by these planes. Then Lemma 11 guarantees that $f(U)$ is spanned by all lines belonging to (1). This means that (1) cannot be contained in a top and we get the claim.

Let $U$ and $T$ be distinct elements of $\mathcal{A}$ containing $p_{i}$. Lemma 12 implies the existence of points $q_{i}(U), q_{i}(T)$ and maximal singular subspaces $U^{\prime}, T^{\prime}$ such that

$$
g\left(\mathcal{A}_{1} \cap\left[p_{i}, U\right]_{1}\right) \subset\left[q_{i}(U), U^{\prime}\right]_{1}
$$

and

$$
g\left(\mathcal{A}_{1} \cap\left[p_{i}, T\right]_{1}\right) \subset\left[q_{i}(T), T^{\prime}\right]_{1} .
$$

If $U$ and $T$ are adjacent vertices of the graph $\Gamma_{\delta}(\Pi)$ then

$$
\operatorname{dim}(U \cap T)=n-3 \geqslant 3
$$

and

$$
\left(\mathcal{A}_{1} \cap\left[p_{i}, U\right]_{1}\right) \cap\left(\mathcal{A}_{1} \cap\left[p_{i}, T\right]_{1}\right)
$$


contains more than one element. The same holds for

$$
\left[q_{i}(U), U^{\prime}\right]_{1} \cap\left[q_{i}(T), T^{\prime}\right]_{1}
$$

(since the restriction of $g$ to every star is injective). This means that $q_{i}(U)=q_{i}(T)$ (if $q_{i}(U)$ and $q_{i}(T)$ are distinct then the latter intersection contains at most one element). The graph $\Gamma_{\delta}(\Pi)$ is connected and the equality $q_{i}(U)=q_{i}(T)$ holds for any $U, T \in \mathcal{A}$ containing $p_{i}$.

Since every line $p_{i} p_{j}$ is contained in a certain $U \in \mathcal{A}$, we get the following: for every point $p_{i}$ there is a point $q_{i}$ such that

$$
g\left(\mathcal{A}_{1} \cap\left[p_{i}\right\rangle_{1}\right) \subset\left[q_{i}\right\rangle_{1} .
$$

The point $q_{i}$ is the intersection of all lines $L_{i j}$.

We show that $q_{i} \neq q_{j}$ if $i \neq j$. The lines $L_{i s}$ and $L_{j t}$ contain $q_{i}$ and $q_{j}$ (respectively) and, by Lemma 8, these lines are disjoint if $\{i, s\} \cap\{j, t\}=\emptyset$.

Thus $L_{i j}$ is spanned by $q_{i}$ and $q_{j}$. Then for every $U \in \mathcal{A}$ the subspace $f(U)$ is spanned by all $q_{i}$ such that $p_{i} \in U$ (see the proof of Lemma 12). So, the required statement is a simple consequence of the following.

Lemma 13. The points $q_{1}, \ldots, q_{2 n}$ form a frame of $\Pi$.

To prove Lemma 13 we need some elementary properties of collinearity relation in polar spaces.

Lemma 14. The following assertions are fulfilled:

(1) if $p \in P$ and $X \subset P$ then $p \perp X$ implies that $p \perp\langle X\rangle$,

(2) if $p \in P$ and $S$ is a maximal singular subspace then $p \perp S$ implies that $p \in S$.

Proof. See, for example, [10, Section 4.1].

Proof of Lemma 13. We have $q_{i} \perp q_{j}$ if $j \neq \sigma(i)$ (since these points span the line $L_{i j}$ ). Suppose that $q_{i} \perp q_{\sigma(i)}$ for a certain $i$. Then $q_{\sigma(i)}$ is collinear to all points $q_{j}$. Every element of $\mathcal{X}$ is spanned by some $q_{j}$ and the first statement of Lemma 14 guarantees that $q_{\sigma(i)} \perp S$ for every $S \in \mathcal{X}$. By the second statement of Lemma 14, every element of $\mathcal{X}$ contains $q_{\sigma(i)}$ and any two elements of $\mathcal{X}$ have a non-empty intersection. The latter contradicts the fact that $\mathcal{X}$ is the image of an isometric embedding of $\frac{1}{2} H_{n}$ in $\Gamma_{\delta}(\Pi)$.

\section{Conjecture}

In this section we will suppose that $\Pi=(P, \mathcal{L})$ is a polar space of type $\mathrm{D}_{n}$, where $n$ is an odd integer greater than 4 . Let $\mathcal{A}$ be the apartment of $\mathcal{G}_{\delta}(\Pi), \delta \in\{+,-\}$ defined by a frame $\mathcal{F}=\left\{p_{1}, \ldots, p_{2 n}\right\}$. Let also $f: \mathcal{A} \rightarrow \mathcal{G}_{\delta}(\Pi)$ be an isometric embedding of $\frac{1}{2} H_{n}$ 
in $\Gamma_{\delta}(\Pi)$. For every $i \in\{1, \ldots, 2 n\}$ the restriction of $\Gamma_{\delta}(\Pi)$ to $\mathcal{A} \cap\left[p_{i}\right\rangle_{\delta}$ is isomorphic to $\frac{1}{2} H_{n-1}$ and Lemma 7 implies the existence of a point $q_{i}$ such that

$$
f\left(\mathcal{A} \cap\left[p_{i}\right\rangle_{\delta}\right) \subset\left[q_{i}\right\rangle_{\delta}
$$

We can prove that there are precisely the following possibilities:

(1) all $q_{i}$ are coincident,

(2) all $q_{i}$ are mutually distinct.

In the second case, an easy verification shows that all $q_{i}$ form a frame and $f(\mathcal{A})$ is the apartment of $\mathcal{G}_{\delta}(\Pi)$ associated to this frame.

We conjecture that the first possibility is realized, i.e. there exists an isometric embedding of $\frac{1}{2} H_{n}$ in $\Gamma_{\delta}(\Pi)$ whose image is not an apartment of $\mathcal{G}_{\delta}(\Pi)$.

In the first case, $f$ can be considered as an isometric embedding of $\frac{1}{2} H_{n}$ in $\Gamma_{\delta}\left(\Pi_{q}\right)$, where $q=q_{1}=\cdots=q_{2 n}$. The rank of the polar space $\Pi_{q}$ is equal to $n-1$. Thus the graphs $\frac{1}{2} H_{n}$ and $\Gamma_{\delta}\left(\Pi_{q}\right)$ are of the same diameter $\frac{n-1}{2}$. This trivial observation supports our conjecture.

\section{Isometric embeddings of half-spin Grassmann graphs}

\subsection{Application of Theorem 2}

Let $\Pi=(P, \mathcal{L})$ and $\Pi^{\prime}=\left(P^{\prime}, \mathcal{L}^{\prime}\right)$ be polar spaces of rank $n$ and $n^{\prime}$, respectively. The existence of isometric embeddings of $\Gamma_{n-1}(\Pi)$ in $\Gamma_{n^{\prime}-1}\left(\Pi^{\prime}\right)$ implies that the diameter of $\Gamma_{n-1}(\Pi)$ is not greater than the diameter of $\Gamma_{n^{\prime}-1}\left(\Pi^{\prime}\right)$, i.e. $n \leqslant n^{\prime}$.

First, we consider the case when $n=n^{\prime}$. Let $f: P \rightarrow P^{\prime}$ be a mapping which transfers every frame of $\Pi$ to a frame of $\Pi^{\prime}$. By [10, Subsection 4.9.6], $f$ sends lines of $\Pi$ to subsets contained in lines of $\Pi^{\prime}$; moreover, if $S$ is a singular subspace of $\Pi$ then $f(S)$ spans a singular subspace of $\Pi^{\prime}$ whose dimension is equal to the dimension of $S$. The mapping which transfers every $X \in \mathcal{G}_{n-1}(\Pi)$ to $\langle f(X)\rangle$ is an isometric embedding of $\Gamma_{n-1}(\Pi)$ in $\Gamma_{n-1}\left(\Pi^{\prime}\right)$. If $\Pi$ and $\Pi^{\prime}$ both are polar spaces of type $D_{n}$ then the restriction of this mapping to $\mathcal{G}_{\delta}(\Pi), \delta \in\{+,-\}$ is an isometric embedding of $\Gamma_{\delta}(\Pi)$ in $\Gamma_{\gamma}\left(\Pi^{\prime}\right), \gamma \in\{+,-\}$.

Suppose that $n \leqslant n^{\prime}$ and $M$ is an $\left(n^{\prime}-n-1\right)$-dimensional singular subspace of $\Pi^{\prime}$. Then $\Pi_{M}^{\prime}$ is a polar space of rank $n$ (if $n=n^{\prime}$ then $M=\emptyset$ and $\Pi_{M}^{\prime}$ coincides with $\Pi^{\prime}$ ). Every frame preserving mapping of $\Pi$ to $\Pi_{M}^{\prime}$ induces an isometric embedding of $\Gamma_{n-1}(\Pi)$ in $\Gamma_{n-1}\left(\Pi_{M}^{\prime}\right)$. This is an isometric embedding of $\Gamma_{n-1}(\Pi)$ in $\Gamma_{n^{\prime}-1}\left(\Pi^{\prime}\right)$, since $\Gamma_{n-1}\left(\Pi_{M}^{\prime}\right)$ can be naturally identified with the restriction of $\Gamma_{n^{\prime}-1}\left(\Pi^{\prime}\right)$ to $[M\rangle_{n^{\prime}-1}$. In the case when $\Pi$ and $\Pi^{\prime}$ are polar spaces of type $\mathrm{D}_{n}$ and $\mathrm{D}_{n^{\prime}}$ (respectively), we get an isometric embedding of $\Gamma_{\delta}(\Pi), \delta \in\{+,-\}$ in $\Gamma_{\gamma}\left(\Pi^{\prime}\right), \gamma \in\{+,-\}$.

Theorem 3 ([12]). Every isometric embedding of $\Gamma_{n-1}(\Pi)$ in $\Gamma_{n^{\prime}-1}\left(\Pi^{\prime}\right)$ is induced by a frame preserving mapping of $\Pi$ to $\Pi_{M}^{\prime}$, where $M$ is an $\left(n^{\prime}-n-1\right)$-dimensional singular subspace of $\Pi^{\prime}$. 
As an application of Theorem 2 we prove the following.

Theorem 4. Suppose that $\Pi$ and $\Pi^{\prime}$ are polar spaces of type $\mathrm{D}_{n}$ and $\mathrm{D}_{n^{\prime}}$, respectively. If $n$ is an even integer not less than 6 then every isometric embedding of $\Gamma_{\delta}(\Pi), \delta \in\{+,-\}$ in $\Gamma_{\gamma}\left(\Pi^{\prime}\right), \gamma \in\{+,-\}$ is induced by a frame preserving mapping of $\Pi$ in $\Pi_{M}^{\prime}$, where $M$ is an $\left(n^{\prime}-n-1\right)$-dimensional singular subspace of $\Pi^{\prime}$.

\subsection{Proof of Theorem 4}

Suppose that $f: \mathcal{G}_{\delta}(\Pi) \rightarrow \mathcal{G}_{\gamma}\left(\Pi^{\prime}\right)$ is an isometric embedding of $\Gamma_{\delta}(\Pi)$ in $\Gamma_{\gamma}\left(\Pi^{\prime}\right)$ and $n$ is even. Then $f$ transfers maximal cliques of $\Gamma_{\delta}(\Pi)$ (stars and special subspaces) to subsets in maximal cliques of $\Gamma_{\gamma}\left(\Pi^{\prime}\right)$.

Lemma 15. The following assertions are fulfilled:

(1) distinct maximal cliques go to subsets of distinct maximal cliques,

(2) the image of every maximal clique of $\Gamma_{\delta}(\Pi)$ is contained in a unique maximal clique of $\Gamma_{\gamma}\left(\Pi^{\prime}\right)$.

Proof. (1). If $\mathcal{X}$ and $\mathcal{Y}$ are distinct maximal cliques of $\Gamma_{\delta}(\Pi)$ whose images are contained in the same maximal clique of $\Gamma_{\gamma}\left(\Pi^{\prime}\right)$ then there exist non-adjacent vertices $X \in \mathcal{X}$ and $Y \in \mathcal{Y}$ such that $f(X)$ and $f(Y)$ are adjacent vertices of $\Gamma_{\gamma}\left(\Pi^{\prime}\right)$. This contradicts the fact that $f$ is an embedding.

(2). Let $\mathcal{C}$ be a maximal clique of $\Gamma_{\delta}(\Pi)$ and let $S$ be the associated singular subspace of $\Pi$ (an element of $\mathcal{G}_{n-4}(\Pi)$ or $\mathcal{G}_{-\delta}(\Pi)$ ). By [10, Proposition 4.7], there is a frame $\mathcal{F}$ such that $S$ is spanned by a subset of $\mathcal{F}$. Let $\mathcal{A}$ be the apartment of $\mathcal{G}_{\delta}(\Pi)$ defined by $\mathcal{F}$. Then $\mathcal{A} \cap \mathcal{C}$ is a base of the projective space $\mathcal{C}$ (see Subsection 4.2). Theorem 2 states that $f(\mathcal{A})$ is an apartment of $\mathcal{G}_{\delta}\left(\Pi_{M}^{\prime}\right)$, where $M$ is an $\left(n^{\prime}-n-1\right)$-dimensional singular subspace of $\Pi^{\prime}$. This guarantees that $f(\mathcal{A} \cap \mathcal{C})$ is an independent subset in every maximal clique of $\Gamma_{\gamma}\left(\Pi^{\prime}\right)$ containing $f(\mathcal{C})$ and the dimension of the subspace spanned by $f(\mathcal{A} \cap \mathcal{C})$ is not less than 3 .

Now suppose that $f(\mathcal{C})$ is contained in two distinct maximal cliques of $\Gamma_{\gamma}\left(\Pi^{\prime}\right)$. If these cliques are of the same type then $f(\mathcal{C})$ is contained in a line of $\mathcal{G}_{\gamma}\left(\Pi^{\prime}\right)$. If one of the cliques is a star and the other is a special subspace then $f(\mathcal{C})$ spans a proper subspace in the star and the dimension of this subspace is not greater than 2. Each of these possibilities contradicts the fact established above.

Lemma 16. One of the following possibilities is realized:

(A) stars go to subsets of stars and special subspaces go to subsets of special subspaces,

(B) stars go to subsets of special subspaces and special subspaces go to subsets of stars.

The second possibility can be realized only in the case when $n=4$. 
Proof. Let $\mathcal{C}$ and $\mathcal{C}^{\prime}$ be maximal cliques of $\Gamma_{\delta}(\Pi)$ such that $\mathcal{C} \cap \mathcal{C}^{\prime}$ is a plane. Then one of these cliques is a star $[M\rangle_{\delta}, M \in \mathcal{G}_{n-4}(\Pi)$ and the other is a special subspace $[U]_{\delta}$, $U \in \mathcal{G}_{-\delta}(\Pi)$. There is a frame $\mathcal{F}$ such that $M$ and $U$ are spanned by subsets of $\mathcal{F}[10$, Proposition 4.7]. Let $\mathcal{A}$ be the apartment of $\mathcal{G}_{\delta}(\Pi)$ defined by $\mathcal{F}$. Then

$$
\mathcal{A} \cap \mathcal{C} \cap \mathcal{C}^{\prime}
$$

is a base of the plane $\mathcal{C} \cap \mathcal{C}^{\prime}$. By Theorem 2, $f(\mathcal{A})$ is an apartment of $\mathcal{G}_{\delta}\left(\Pi_{M}^{\prime}\right)$, where $M$ is an $\left(n^{\prime}-n-1\right)$-dimensional singular subspace of $\Pi^{\prime}$. This means that

$$
f\left(\mathcal{A} \cap \mathcal{C} \cap \mathcal{C}^{\prime}\right)
$$

is an independent subset in the intersection of the maximal cliques of $\Gamma_{\gamma}\left(\Pi^{\prime}\right)$ containing $f(\mathcal{C})$ and $f\left(\mathcal{C}^{\prime}\right)$. Since this set contains three elements, the dimension of the intersection is not less than 2. Therefore, the intersection of the maximal cliques of $\Gamma_{\gamma}\left(\Pi^{\prime}\right)$ containing $f(\mathcal{C})$ and $f\left(\mathcal{C}^{\prime}\right)$ is a plane.

Lemma 15 implies that $f$ induces an injective mapping of the set of maximal cliques of $\Gamma_{\delta}(\Pi)$ to the set of maximal cliques of $\Gamma_{\gamma}\left(\Pi^{\prime}\right)$. Using Lemma 3, we show that one of the possibilities $(\mathrm{A})$ or $(\mathrm{B})$ is realized.

Now suppose that $n \geqslant 6$. Let $U \in \mathcal{G}_{-\delta}(\Pi)$. We take a frame $\mathcal{F}$ such that $U$ is spanned by a subset of $\mathcal{F}$ and denote by $\mathcal{A}$ the associated apartment of $\mathcal{G}_{\delta}(\Pi)$. Then $\mathcal{A} \cap[U]_{\delta}$ is a base of the projective space $[U]_{\delta}$. As above, we establish that $f\left(\mathcal{A} \cap[U]_{\delta}\right)$ is an independent subset in the maximal clique of $\Gamma_{\gamma}\left(\Pi^{\prime}\right)$ containing $f\left([U]_{\delta}\right)$. Since $f\left(\mathcal{A} \cap[U]_{\delta}\right)$ consists of $n$ elements and $n>4$, the maximal clique containing $f\left([U]_{\delta}\right)$ cannot be a star and the possibility $(\mathrm{B})$ is not realized.

From this moment we suppose that $f$ satisfies (A). Then for every $U \in \mathcal{G}_{-\delta}(\Pi)$ there is unique $U^{\prime} \in \mathcal{G}_{-\gamma}\left(\Pi^{\prime}\right)$ such that

$$
f\left([U]_{\delta}\right) \subset\left[U^{\prime}\right]_{\gamma}
$$

We set $f(U):=U^{\prime}$ and extend $f$ to a mapping of $\mathcal{G}_{n-1}(\Pi)$ to $\mathcal{G}_{n^{\prime}-1}\left(\Pi^{\prime}\right)$. The first part of Lemma 15 guarantees that the extension is injective.

Lemma 17. The following assertions are fulfilled:

(1) $f$ transfers adjacent vertices of $\Gamma_{n-1}(\Pi)$ to adjacent vertices of $\Gamma_{n^{\prime}-1}\left(\Pi^{\prime}\right)$,

(2) $d(X, Y)=d(f(X), f(Y))$ if $X, Y \in \mathcal{G}_{\delta}(\Pi)$.

Proof. (1). If $X \in \mathcal{G}_{\delta}(\Pi)$ and $Y \in \mathcal{G}_{-\delta}(\Pi)$ are adjacent vertices of $\Gamma_{n-1}(\Pi)$ then $X$ belongs to $[Y]_{\delta}$ and

$$
f(X) \in[f(Y)]_{\gamma}
$$

which implies that $f(X)$ and $f(Y)$ are adjacent vertices of $\Gamma_{n^{\prime}-1}\left(\Pi^{\prime}\right)$.

(2). This follows from the fact that the restriction of $f$ to $\mathcal{G}_{\delta}(\Pi)$ is an isometric embedding of $\Gamma_{\delta}(\Pi)$ in $\Gamma_{\gamma}\left(\Pi^{\prime}\right)$. 
By Theorem 3, it is sufficient to show that $f$ is an isometric embedding of $\Gamma_{n-1}(\Pi)$ in $\Gamma_{n^{\prime}-1}\left(\Pi^{\prime}\right)$. Since for any two distinct elements of $\mathcal{G}_{n-1}(\Pi)$ there is an apartment containing them [10, Proposition 4.7], we need to show that the restriction of $f$ to every apartment of $\mathcal{G}_{n-1}(\Pi)$ is an isometric embedding of $H_{n}$ in $\Gamma_{n^{\prime}-1}\left(\Pi^{\prime}\right)$.

Let $\mathcal{A}$ be an apartment of $\mathcal{G}_{n-1}(\Pi)$. Then

$$
\mathcal{A}_{\delta}:=\mathcal{A} \cap \mathcal{G}_{\delta}(\Pi)
$$

is an apartment of $\mathcal{G}_{\delta}(\Pi)$ and $\mathcal{A} \backslash \mathcal{A}_{\delta}$ is an apartment of $\mathcal{G}_{-\delta}(\Pi)$. Every special subset of $\mathcal{A}_{\delta}$ is defined by an element of $\mathcal{A} \backslash \mathcal{A}_{\delta}$, i.e. it is the intersection of $\mathcal{A}_{\delta}$ and $[U]_{\delta}$, where $U \in \mathcal{A} \backslash \mathcal{A}_{\delta}$. Theorem 2 states that $f\left(\mathcal{A}_{\delta}\right)$ is an apartment of $\mathcal{G}_{\gamma}\left(\Pi_{M}^{\prime}\right)$, where $M$ is an $\left(n^{\prime}-n-1\right)$-dimensional singular subspace of $\Pi^{\prime}$. Let $\mathcal{A}^{\prime}$ be the apartment of $\mathcal{G}_{n-1}\left(\Pi_{M}^{\prime}\right)$ containing $f\left(\mathcal{A}_{\delta}\right)$. Then $\mathcal{A}^{\prime} \backslash f\left(\mathcal{A}_{\delta}\right)$ is an apartment of $\mathcal{G}_{-\gamma}\left(\Pi_{M}^{\prime}\right)$ and every special subset of $f\left(\mathcal{A}_{\delta}\right)$ is defined by an element of $\mathcal{A}^{\prime} \backslash f\left(\mathcal{A}_{\delta}\right)$. Since $f$ transfers special subsets of $\mathcal{A}_{\delta}$ to special subsets of $f\left(\mathcal{A}_{\delta}\right)$, we have

$$
f\left(\mathcal{A} \backslash \mathcal{A}_{\delta}\right)=\mathcal{A}^{\prime} \backslash f\left(\mathcal{A}_{\delta}\right)
$$

which implies that $f(\mathcal{A})=\mathcal{A}^{\prime}$.

Now we show that

$$
d(X, Y)=d(f(X), f(Y))
$$

for all $X, Y \in \mathcal{A}$. By the second part of Lemma 17, this is true if $X, Y \in \mathcal{A}_{\delta}$. In the case when $X \in \mathcal{A} \backslash \mathcal{A}_{\delta}$ and $Y \in \mathcal{A}_{\delta}$, we have $d(X, Y) \leqslant n-1$ and there is a geodesic $Y_{0}, Y_{1}, \ldots, Y_{i}$ in $\Gamma_{n-1}(\Pi)$ such that

$$
Y_{0} \in \mathcal{A}_{\delta}, \quad Y_{1}=X, \quad Y_{i}=Y
$$

Lemma 17 shows that

$$
d\left(Y_{0}, Y_{i}\right)=d\left(f\left(Y_{0}\right), f\left(Y_{i}\right)\right)
$$

and

$$
f\left(Y_{0}\right), f\left(Y_{1}\right), \ldots, f\left(Y_{i}\right)
$$

is a geodesic in $\Gamma_{n^{\prime}-1}\left(\Pi^{\prime}\right)$ which implies (2). If $X, Y \in \mathcal{A} \backslash \mathcal{A}_{\delta}$ and $d(X, Y) \leqslant n-2$ then there is a geodesic $Y_{0}, Y_{1}, \ldots, Y_{i}$ in $\Gamma_{n-1}(\Pi)$ such that

$$
Y_{0}, Y_{i} \in \mathcal{A}_{\delta}, \quad Y_{1}=X \quad Y_{i-1}=Y .
$$

As above, the image of this geodesic is a geodesic in $\Gamma_{n^{\prime}-1}(\Pi)$ and we get (2).

Consider the case when $X, Y \in \mathcal{A} \backslash \mathcal{A}_{\delta}$ are opposite vertices of $\Gamma_{n-1}(\Pi)$. It is clear that

$$
\mathcal{A} \backslash\{Y\}=\{Z \in \mathcal{A}: d(X, Z) \leqslant n-1\}
$$

There is unique $Y^{\prime} \in \mathcal{A}^{\prime}$ satisfying $d\left(f(X), Y^{\prime}\right)=n$ and

$$
\mathcal{A}^{\prime} \backslash\left\{Y^{\prime}\right\}=\left\{Z^{\prime} \in \mathcal{A}^{\prime}: d\left(f(X), Z^{\prime}\right) \leqslant n-1\right\} .
$$


It follows from the above arguments that

$$
f(\mathcal{A} \backslash\{Y\})=\mathcal{A}^{\prime} \backslash\left\{Y^{\prime}\right\}
$$

Then $f(Y)=Y^{\prime}\left(\right.$ since $\left.f(\mathcal{A})=\mathcal{A}^{\prime}\right)$ and $(2)$ holds.

So, we get the following.

Proposition 1. If $\Pi$ and $\Pi^{\prime}$ are polar spaces of type $\mathrm{D}_{n}$ and $\mathrm{D}_{n^{\prime}}$ (respectively) and $n$ is even then every isometric embedding of $\Gamma_{\delta}(\Pi), \delta \in\{+,-\}$ in $\Gamma_{\gamma}\left(\Pi^{\prime}\right), \gamma \in\{+,-\}$ satisfying (A) is induced by a frame preserving mapping of $\Pi$ in $\Pi_{M}^{\prime}$, where $M$ is an $\left(n^{\prime}-n-1\right)$-dimensional singular subspace of $\Pi^{\prime}$.

Theorem 4 is a direct consequence of Proposition 1 and Lemma 16.

\subsection{Remarks on the case $n=4$}

Suppose that $\Pi$ is a polar space of type $D_{4}$. Then the associated half-spin Grassmannians (together with the families of lines) also are polar spaces of type $D_{4}$. These polar spaces are denoted by $\Pi_{+}$and $\Pi_{-}$(Example 2 ).

Every maximal singular subspace of the polar space $\Pi_{\delta}, \delta \in\{+,-\}$ is a star (defined by a point of $\Pi$ ) or a special subspace (associated to an element of $\mathcal{G}_{-\delta}(\Pi)$ ). Using the intersection properties given in Section 4.1, we can show that one of the half-spin Grassmannians of $\Pi_{\delta}$ consists of all stars and the other is formed by all special subspaces. The corresponding polar spaces can be identified with $\Pi$ and $\Pi_{-\delta}$. By Remark 2, they are isomorphic.

So, the polar spaces $\Pi$ and $\Pi_{\delta}, \delta \in\{+,-\}$ are isomorphic. Every collineation between $\Pi$ and $\Pi_{-\delta}$ induces a collineation of $\Pi_{\delta}$ to itself (an automorphism of $\Gamma_{\delta}(\Pi)$ ) which transfers stars to special subspaces and special subspaces to stars.

Let $f$ be an isometric embedding of $\Gamma_{\delta}(\Pi), \delta \in\{+,-\}$ in $\Gamma_{\gamma}\left(\Pi^{\prime}\right), \gamma \in\{+,-\}$ satisfying (B). We take any automorphism $h$ of $\Gamma_{\delta}(\Pi)$ sending stars to special subspaces and special subspaces to stars. Then $f h$ is an isometric embedding of $\Gamma_{\delta}(\Pi)$ in $\Gamma_{\gamma}\left(\Pi^{\prime}\right)$ satisfying $(\mathrm{A})$ and we can apply Proposition 1.

\subsection{Remarks on frame preserving mappings}

In this subsection we suppose that $\Pi=(P, \mathcal{L})$ and $\Pi^{\prime}=\left(P^{\prime}, \mathcal{L}^{\prime}\right)$ are polar spaces of an arbitrary type and embedable in the projective spaces $\Pi_{V}$ and $\Pi_{V^{\prime}}$ associated to vector spaces $V$ and $V^{\prime}$, respectively. It is well-known that every collineation of $\Pi$ to $\Pi^{\prime}$ can be extended to a collineation of $\Pi_{V}$ to $\Pi_{V^{\prime}}$ and, by the Fundamental Theorem of Projective Geometry, the latter collineation is induced by a semilinear isomorphism of $V$ to $V^{\prime}[5$, Chapter III].

Let $f: P \rightarrow P^{\prime}$ be a mapping which transfers frames of $\Pi$ to frames of $\Pi^{\prime}$. It was noted above that this mapping sends lines of $\Pi$ to subsets in lines of $\Pi^{\prime}$. We state that $f$ can be extended to a mapping of $\Pi_{V}$ to $\Pi_{V^{\prime}}$ which transfers lines of $\Pi_{V}$ to subsets in lines of $\Pi_{V^{\prime}}$ (the proof is a modification of the proof given in $[5$, Chapter III]). It follows from 
a generalized version of the Fundamental Theorem of Projective Geometry $[6,7]$ that the latter mapping is induced by a semilinear mapping $l: V \rightarrow V^{\prime}$ (it must be pointed out that the associated homomorphism between the division rings is not necessarily surjective). Also, we state that the mapping $l: V \rightarrow V^{\prime}$ is a strong semilinear embedding, i.e. it transfers any collection of linearly independent vectors to linearly independent vectors. In some special cases, for example, if our polar spaces are of type $\mathrm{D}_{n}$, or for the symplectic polar spaces, this statement is obvious. In the general case, we need some algebraic technique to prove it. For this reason, it will be natural to consider this question in a separate paper.

\section{Acknowledgements}

The author is grateful to the referee for a question concerning frame preserving mappings.

\section{References}

[1] F. Buekenhout, A. M. Cohen. Diagram geometry. Ergebnisse der Mathematik und ihrer Grenzgebiete/Results in Mathematics and Related Areas, 57. Springer, Heidelberg, 2013.

[2] B. N. Cooperstein, E. E. Shult. Frames and bases of Lie incidence geometries. J. Geom., 60:17-46, 1997.

[3] B. N. Cooperstein, A. Kasikova, E. E. Shult. Witt-type Theorems for Grassmannians and Lie Incidence Geometries. Adv. Geom., 5:15-36, 2005.

[4] B. N. Cooperstein. Witt-Type theorems for subspaces of Lie geometries: A survey. In Groups of Exceptional Type, Coxeter Groups and Related Geometries. Springer Proceedings in Mathematics and Statistics, 82:123-133, 2013.

[5] J. Dieudonné. La géométrie des groupes classiques. Springer, New York-HeidelbergBerlin, 1971.

[6] C. A. Faure, A. Frölicher. Morphisms of projective geometries and semilinear maps. Geom. Dedicata, 53:237-262, 1994.

[7] H. Havlicek. A generalization of Brauner's theorem on linear mappings. Mitt. Math. Sem. Univ. Giessen, 215:27-41, 1994.

[8] A. Kasikova. Characterization of some subgraphs of point-collinearity graphs of building geometries. European J. Combin., 28:1493-1529, 2007.

[9] A. Kasikova. Characterization of some subgraphs of point-collinearity graphs of building geometries II, Adv. Geom., 9:45-84, 2009.

[10] M. Pankov. Grassmannians of classical buildings. Algebra and Discrete Math. Series, no. 2. World Scientific, Singapore, 2010.

[11] M. Pankov. Isometric embeddings of Johnson graphs in Grassmann graphs. J. Algebraic Combin., 33:555-570, 2011. 
[12] M. Pankov. Metric characterization of apartments in dual polar spaces. J. Combin. Theory Ser. A, 118:1313-1321, 2011.

[13] M. Pankov. Characterization of apartments in polar Grassmannians. Bull. Belg. Math. Soc. Simon Stevin, 19:345-366, 2012.

[14] A. Pasini. Diagram Geometries. Oxford Science Publications. Clarendon/Oxford University Press, New York, 1994.

[15] E. E. Shult. Points and Lines. Characterizing the Classical Geometries. Universitext. Springer, Berlin, 2011.

[16] J. Ueberberg. Foundations of incidence geometry. Projective and polar spaces. Springer Monographs in Mathematics. Springer, Heidelberg-Berlin, 2011.

[17] J. Tits. Buildings of spherical type and finite BN-pairs. Lecture Notes in Mathematics, 386. Springer-Verlag, Berlin-New York, 1974. 